\title{
Physical, Mechanical and Antimicrobial Evaluations of Physically Crosslinked PVA/Chitosan Hydrogels Containing Nanoparticles
}

\author{
Ahmed Saad Montaser \\ Preparation and Finishing Department, Textile,m Research, Division, National Research Centre, Dokki, Giza, Egypt.
}

\section{ARTICLE INFO \\ Article history: \\ Received on: 10/12/2015 \\ Revised on: 23/01/2016 \\ Accepted on: 16/02/2016 \\ Available online: 28/05/2016}

Key words:

PVA, Chitosan, Freeze-

thawing, AgNPs, MMT,

hydrogel, Antibacterial.

\begin{abstract}
Chitosan/montmorillonite (MMT) and Poly vinyl Alcohol (PVA) / Silver nanoparticles (AgNPs) nanocomposites were prepared and formed hydrogel membranes using freeze thawing technique. The properties of the prepared hydrogels were investigated and compared to hydrogel membranes in presence and absence of nanometals. The physical behavior, mechanical properties and antibacterial activity was examined. Also the surface morphology monitored using scanning electron microscope. Antimicrobial activity against bacteria and yeast was also examined. The obtained results showed positive effect of nanometals especially AgNPs on swelling percent on the other hand tensile strength were combined by presence of MMT nanoparticles. The surface morphology showed homogenous images for all samples except samples containing MMT. All prepared samples containing nanoparticles showed antibacterial activity especially hydrogel membranes containing AgNPs.
\end{abstract}

\section{INTRODUCTION}

Hydrogel identified as 3D hydrophilic polymer network can hold the water molecules for a long time preventing them from evaporation. Hydrogel widely used in medical industry such as wound dressing, scaffold, contact lenses, controlled drug release and etc (Bhowmick and Koul, 2016; Catanzano et al., 2015; Silva et al., 2015; Pairatwachapun et al., 2016; Mabry et al., 2015). Hydrogels prepared in physical and chemical ways. Physical ways especially freeze thawing techniques it has advantageous of easy prepare and free from harmful initiators and crosslinkers which not easy to remove (Abdel-Mohsen et al., 2011). Hydrogels prepared from PVA aqueous solutions by freeze-thawing have shown interesting properties (Kim et al., 2015). They have good mechanical properties and stable thermal properties (Kim et al., 2015). Unfortunately, PVA showed high crosslinking at pure state affected negatively on swell-ability. For this reason, freeze-thawed PVA hydrogel usually incorporated with another hydrophilic polymer (Zhang et al., 2010).

\footnotetext{
* Corresponding Author

Email:dr_asaadnrc@yahoo.com, Phone No: +201144409443
}

Chitosan, the partially deacetylated from chitin, is well known material for wound healing field. It has excellent biodegradability, biocompatibility and antibacterial activity (Abdel-Mohsen et al., 2012; Hebeish et al., 2014; Hebeish et al., 2015; Hebeish et al., 2013).

Now a days chitosan incorporated to different nanometals to quire it new properties such as mechanical strength(Hsu S-H et al., 2012). In the current work chitosan/MMT and PVA/AgNPs are prepared to form physical hydrogel using freeze-thawing technique studying the effect of presence and absence nanometals of physical, mechanical and antibacterial properties.

\section{EXPERIMENTAL}

\section{Material and methods}

Materials

PVA powder of molecular weight $17 \mathrm{kDa}$ with saponification degree of $99.85 \%$ was supplied by D-MID GEL Co. Koysna, Monfiya, Egypt. Chitosan powder was supplied by Fluka Chemical, and its degree of acetylation was determined and found to be $14 \%$ by elementary analysis with average molecular weight $600 \mathrm{KDa}$. Silver nitrate and montmorillonite were laboratory grade used without further purification. 


\section{Methods}

Preparation of silver nanoparticles (AgNPs) by polyvinyl alcohol and sodium borohydride.

Ten grams of PVA was dissolved in $100 \mathrm{ml}$ distilled water using heating magnetic stirrer at $70{ }^{\circ} \mathrm{C}$ for $6 \mathrm{hr}$. After complete dissolution, $5 \mathrm{mg}$ sodium borohydride $\left(\mathrm{NaBH}_{4}\right)$ was added to the reaction medium followed by drop-wise addition of $10 \mathrm{mg} / \mathrm{ml}$ of silver nitrate solution (keeping in mind that the total volume of the reaction medium is $100 \mathrm{ml}$ ). The reaction mixture was kept under continuous stirring for $(15 \mathrm{~min})$. The reaction medium acquires a clear yellow color indicating the formation of silver nanoparticles (Bhowmick and Koul, 2016).

\section{Preparation of CS / MMT nanocomposites}

One gram of CS was dissolved $1 \%$ glacial acetic acid and agitated for $24 \mathrm{~h}$. $50 \mathrm{mg}$ of MMT were added in to chitosan solution followed by continuous stirring for $2 \mathrm{hrs}$.

\section{Hydrogel formation}

Freeze thawing technique as mentioned before as a safe effective free crosslinking at hydrogel preparation provide a real solution for biomedical hydrogel prospect to be used at wound healing. Our group the optimum condition for preparing chitosan/PVA freeze-thawing hydrogel, it was as follow, chitosan: PVA composition was (50/50) (W: W) weight by weight, 3 freeze thawing cycles and $24 \mathrm{hr}$ for the cycle time. Herein, we apply the optimum condition for the two polymers (for PVA/AgNPs nancomposites and $\mathrm{Cs} / \mathrm{MMT}$ nancomposites). Table 1 indicates hydrogel membrane compositions which successfully forming elastic and handle hydrogel, the following sections will indicate the effect of nanometals on the physical and mechanical properties of the obtained membrane.

Table 1: Hydrogel nancomposites composition used in membrane formation

\begin{tabular}{llllll}
\hline Sample & PVA & PVA/AgNPs & Cs & Cs/MMT & total \\
\hline PVA/Cs & $50 \%$ & & $50 \%$ & & $100 \%$ \\
PVA/AgNPs: Cs & & $50 \%$ & $50 \%$ & & $100 \%$ \\
PVA:Cs/MMT & $50 \%$ & & & $50 \%$ & $100 \%$ \\
PVA/AgNPs: & & $50 \%$ & & $50 \%$ & $100 \%$ \\
Cs/MMT & & & & & \\
\hline
\end{tabular}

\section{Characterization of the nancomposites}

Ultra Violet-Visible (UV-Vis) Spectra

UV-vis spectral analysis was done by using a 50 ANALYTIKA, JENA Spectrophotometer from 200 to $500 \mathrm{~nm}$.

\section{Transmission Electron Microscope (TEM)}

TEM was used to assess the potential impact of the modification on the elemental and structural properties of the synthesized PVA/AgNPs. The TEM analysis was done usingJEOL-JEM-1200 (Japan).

\section{Scanning Electron Microscope (SEM)}

SEM analysis was done using a scanning electron probe micro analyzer (JXA-840A, Japan). The specimens in the form of films were mounted on the specimen stabs and coated with thin film of gold by the sputtering method. The micrograph was taken at magnification of 1000 using (KV) accelerating voltage. The composition of selected nanoparticles was determined by energy dispersive X-ray (EDX) analysis using INCa (X Sight) (England).

\section{Swelling Percent}

Pre weighed dry samples of the prepared PVA/chitosan hydrogels and PVA/chitosan containing nancomposites as mentioned in table 1. Dried samples were immersed in buffer solutions at $\mathrm{pH} 7$. At $25^{\circ} \mathrm{C}$, the samples were weighed at set intervals 1, 2, 3, 4, 24 and $48 \mathrm{hr}$ and the changes of weight were recorded; before samples were weighed they were blotted to remove any excess media from the surface. The swelling percent was calculated using the following equation 1 :

$S w \%=(W s-W d) / W d \times 100$ equation 1 . where $\mathrm{Sw}(\%)$ is swelling percent, $\mathrm{Wd}$ and $\mathrm{Ws}$ are the weights of the samples in the dry and swollen states respectively (Abureesh et al.,).

\section{Mechanical properties}

The mechanical properties of PVA/Chitosan hydrogel membrane in presence and absence nanomaterials were examined using tensile elongation tests. Typically, equilibrium swollen membranes were cut with $25 \mathrm{~cm}$ length and $5 \mathrm{~cm}$ weidth with fixed thickness. The breaking load of the samples was determined by the strip method on tensile tester instrument (ASANO Machine MFG.CO, DSAKA, No.6202, Japan) according to ASTM method D2256-66T (ASTM Test Method, 1972). Elongation- at-breakwas determined according to ASTM procedure D-2296-66T (Liu et al., 2010).

\section{Antimicrobial Activity Test}

Test disc diffusion method with some modification was used for screening the membrane samples for antimicrobial activity (Ericson and Sherris, 1971). Nutrient agar for bacteria 0.1 $\mathrm{ml}$ of an appropriate dilution of the test culture was used. The prepared hydrogel membranes (1-cm diameter) were placed on the surface of the incubated plates at $35^{\circ} \mathrm{C}$ for 24 hours. Diameter of plates inhibition zone $(\mathrm{mm})$ including the disc diameter was measured for each treatment.

\section{RESULTS AND DISCUSSIONS Preparation of PVA/ AgNPs}

Reduction method one of the famous ways for silver nanoparticles production. Silver salt ionized in solution forming silver cations and complementary anion. Herein our work, NaBH4 used as reducing agent in presence of PVA which acting as cluster stabilizer. Fig. 1a clearly shown the difference of the color between PVA precursor and PVA/AgNPS with the yellow color, These particles at Fig.1b have specific optical properties indicated by the presence of intense absorption band around $400 \mathrm{~nm}$ caused by collective excitation of all the free electrons in the particles. TEM images (Figure1c) are situated that the prepared particles lying in the nanosized range from 20-50 nm (Guo et al., 2013). 

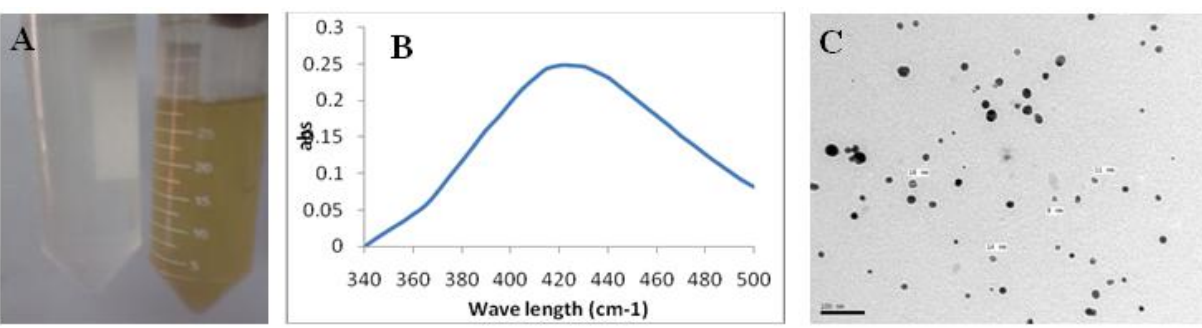

Fig. 1: Nanoparticles formation of PVA/AgNPs; a) Optical image, b) Uv-vis absorpance, c) TEM images.

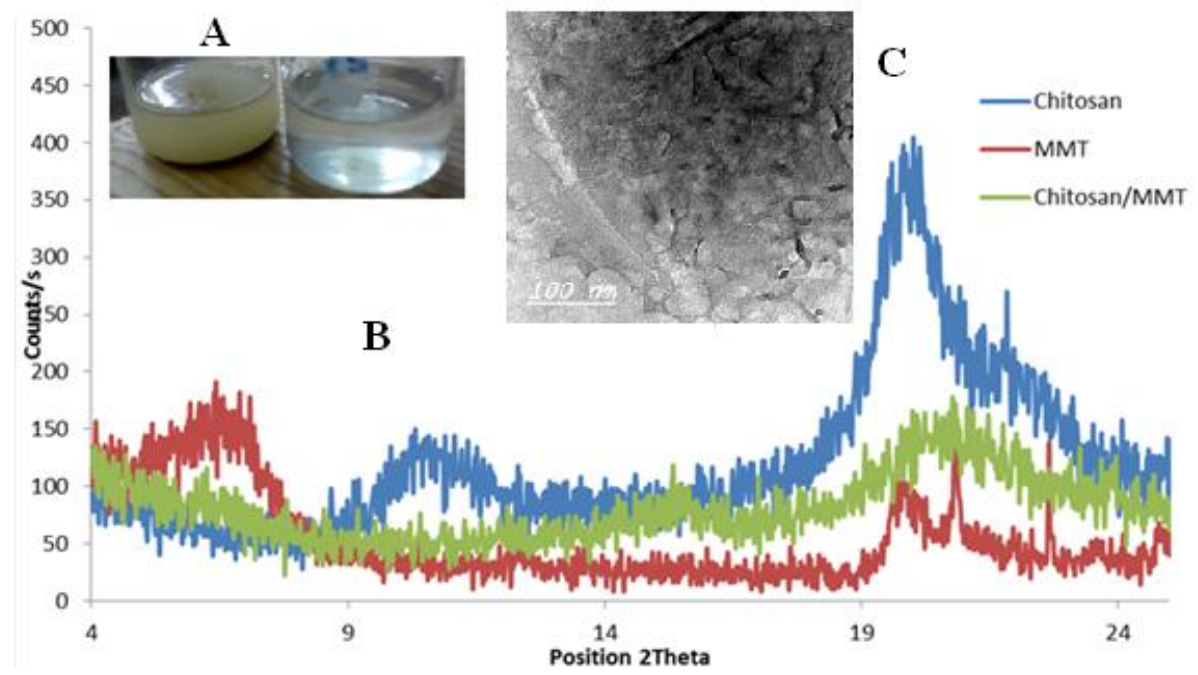

Fig. 2: Cs: MMT nanocomposite formation and characterization. Where a) Optical image, b)XRD of chitosan /MMT nanocomposite and c) TEM images of chitosan /MMT nanocomposite.

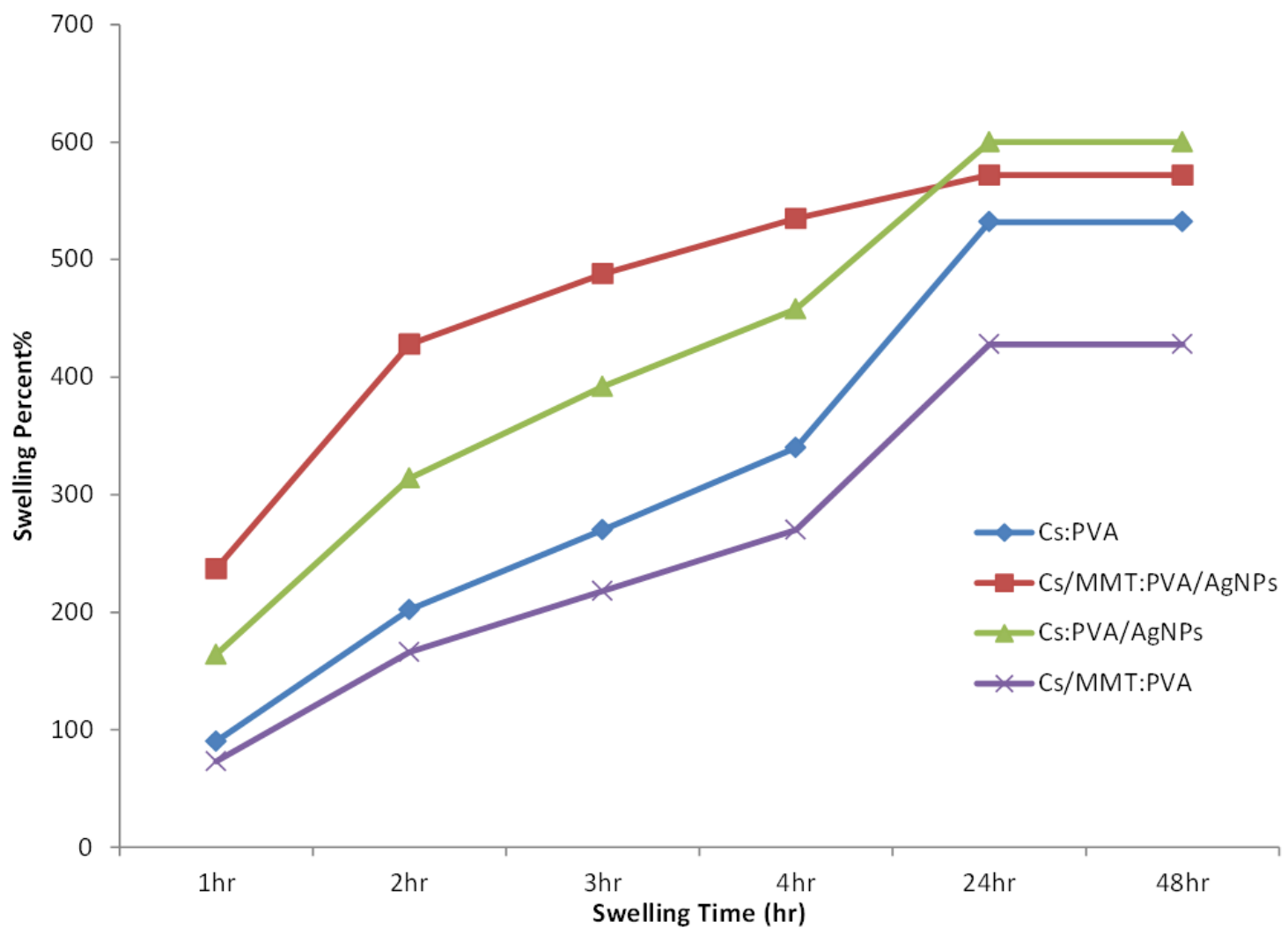

Fig. 3: Swelling percent of PVA freeze thawed membranes. 


\section{Characterization of Exfoliated chitosan / MMT using X-Ray Diffraction (XRD) and Transmission Electron Microscope (TEM)}

Nanocomposites are shown Fig. 2a. It's clearly appearing the difference in color between chitosan and chitosan / MMT nancomposite. Also Fig. $2 \mathrm{~b}$ two clear XRD reflections at $2 \theta=11.6^{\circ}$ and $20^{\circ}$ were observed for CS (Fig. 2b) and the intensity of the chitosan decreases when MMT was added by (1/20). And small three additional reflections appeared at $8.5^{\circ}, 16.1^{\circ}$ and $22.9^{\circ}$. Fig. 2C TEM images (Fig. 2c) of the prepared Cs/MMT nanocomposite shows dispersed layers in nanosized. As published, MMT layers separated with metal ions and chitosan as cationic biopolymer used to exfoliated these layers by substituted the interlayer metal cations (Hsu et al., 2012).

\section{Swelling Percent}

Swelling percent of PVA freeze thawed hydrogel ordinary affected by composition percent, $\mathrm{PH}$, freezing time, number of freezing cycles and time of each cycle etc. in the current paper we study the effect of incorporation nanometals on the prepared chitosan/PVA freeze thaw membrane.

As known swelling steps starts with absorption, diffusion and relaxation. Firstly absorption depends on hydrophilicity of the composition, it is clearly shown at Fig. 3 that membranes containing MMT as hydrophilic nanomaterials showed high swelling percent at first hour. Diffusion step completely depend on network structure. The presence of nanometals affected negatively on the crosslinking capacity and positively on swelling percent.

It's clearly observed that swelling percent of membrane containing two nanometals MMT and AgNPs more than containing only each one of them. In the case of presence of one of the nanometals, it's obvious that MMT with time pass it may separation process occur from chitosan. chitosan amino group preferred attraction to hydroxyl group of PVA which effect on the membrane hydrophilicity and swelling percent, also PVA membranes containing AgNPs showed high swelling due to blocking effect of cationic AgNPs to negative site of PVA hydroxyl groups affecting negatively on crosslinking process positively on swelling percent (Chhatri A et al., 2011).

\section{Mechanical Properties}

For all membranes prepared by freeze thaw cycle, the mechanical properties of the hybride hydrogel membranes better than pure Cs:PVA membrane hydrogel. Tensile strength results Figure4a and maximum loaded fig. 5b showed that MMT played a very good role in the enforcement of hydorgel network, it is possible for PVA to form crystalline coating around the MMT layers and AgNPs, maximizing interfacial stress transfer.

This may be reason why the mechanical properties of the membrane containing MMT are better than the pure Cs: PVA hydrogels. Indeed, originally polymer provides high mechanical provides high elongation. In our case may nanocomposites strengthen internal network and did not provide elasticity to the prepared hydrogel membranes (Tong et al., 2007).

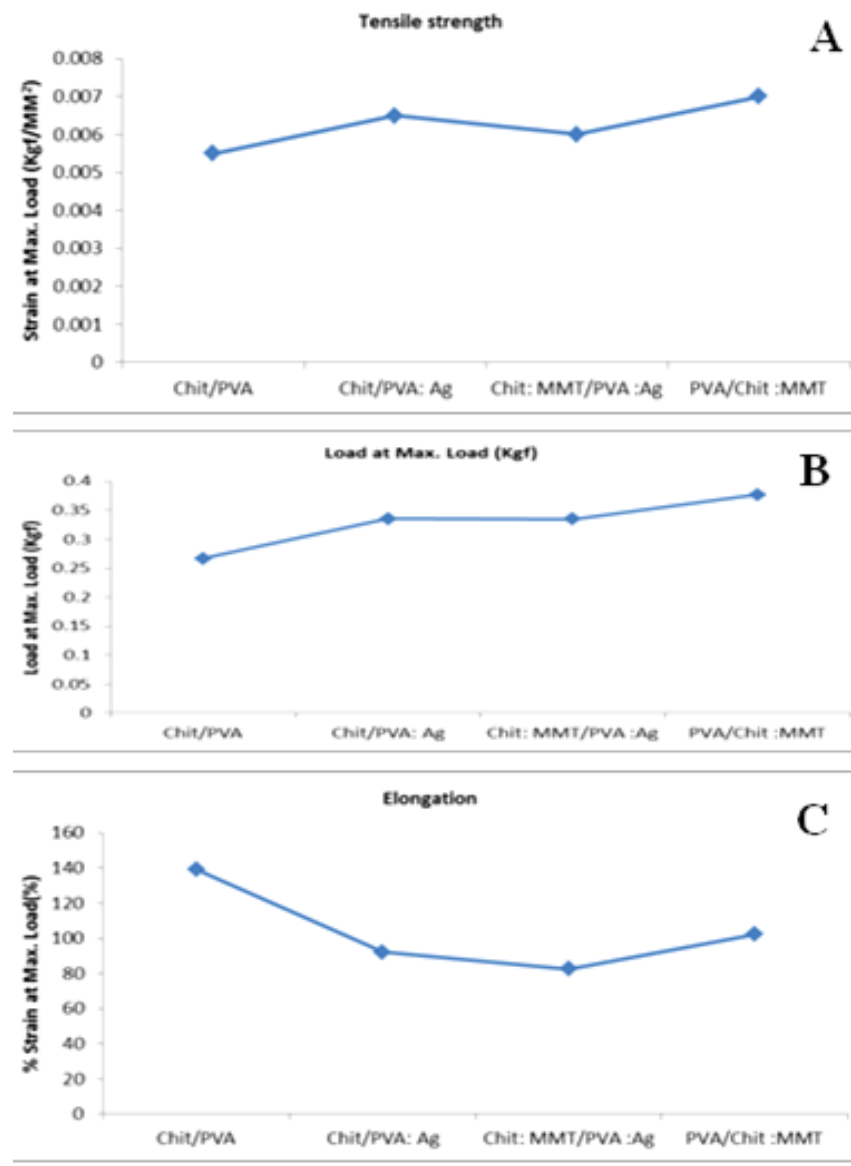

Fig. 4: Mechanical properties of PVA freeze thawed membranes. Where a) Tensile strength, b) force at break and c) Elongation.
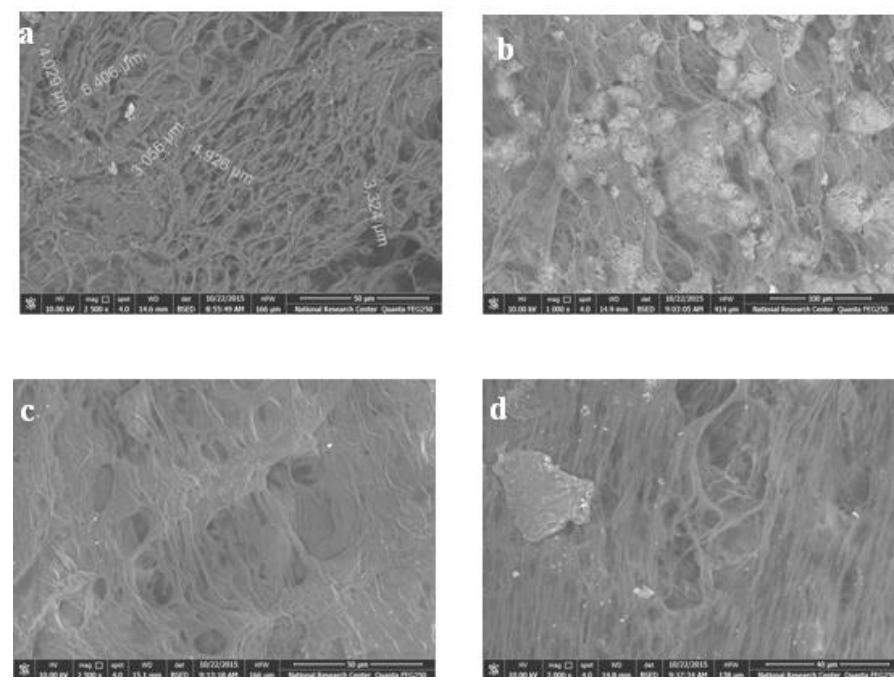

Fig. 5: Scanning electron microscope (SEM) of a) Cs/PVA, b) Cs/ MMT: PVA/AgNPs, c) Cs:PVA/ AgNPs and d) Cs/ MMT:PVA.

\section{Scanning Electron microscope (SEM)}

The surface morphology of the prepared membrane remained similar to Cs: PVA prepared samples; the hydrogel membrane containing MMT has changed to a amorphous morphology. As mentioned, these coagulation presence on the surface Figure5 b,c may resulted from chitosan electrostatic 
attraction to PVA. The presence on MMT on the surface affected positively on the first step of the membrane swelling as mentioned before. Also, homogenous pores and open pores clearly showed to membranes don't containing nanometals over than containing silver nanoparticles and normally over membranes containing MMT. Small pores related to membrane containing nanometals enable an effective capillary attraction provided high swelling percent which understandable us as smaller pore size doesn't mean a smaller porosity.

\section{Antibacterial activity}

The antibacterial activity of the prepared membranes in presence and absence of nanomaterials towards Staphylococcus aureus as a gram-positive coccal bacterium and Pseudomonas as a genus of Gram-negative and Candida as a yeast were examined. The results Figure $6 \mathrm{a}, \mathrm{b}$ and $\mathrm{c}$ and Table 2 showed bacterial growth on the sample don't containing nanometals.

Table 2: Antibacterial activity of the prepared Cs: PVA freeze thawed membranes in presence and absence nanoparticles.

\begin{tabular}{cccc}
\hline & \multicolumn{3}{c}{ Inhibition Zone (mm) } \\
\hline Sample & $\begin{array}{c}\text { Staphylococcus } \\
\text { aureus (Ge+) }\end{array}$ & $\begin{array}{c}\text { Pseudomona } \\
\text { S (Ge-) }\end{array}$ & $\begin{array}{c}\text { Candida } \\
\text { (yeast) }\end{array}$ \\
\hline PVA/Cs & 12 & 11 & 12 \\
PVA/AgNPs: Cs & 14 & 13 & 15 \\
PVA:Cs/MMT & 11 & 10 & 11 \\
PVA/AgNPs: Cs/MMT & 12 & 11 & 12 \\
\hline
\end{tabular}
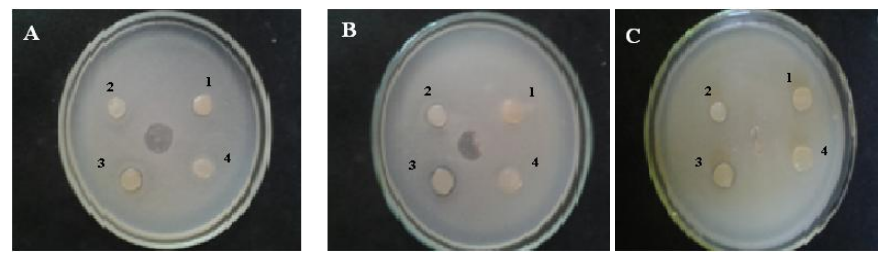

Fig. 6: Antibacterial activity against a) Staphylococcus aureus as a grampositive coccal bacterium, b) Pseudomonas as a genus of Gram-negative and c) Candida as a yeast. Where 1) Cs: PVA/ AgNPs, 2) Cs: PVA, 3) Cs: PVA/ AgNPs and 4) PVA: Cs/ MMT.

All membranes containing AgNPs showed antibacterial activity especially samples containing silver nanoparticles which known with its antibacterial activity (Lavorgna et al., 2014). Also chitosan / PVA membrane shows antibacterial activity thanks to amino group of chitosan which known with its antimicrobial power. Cs/MMT: PVA the week membrane among all samples, it may due to the blocking effect of MMT to the amino group of chitosan which affect negatively on the antibacterial activity.

\section{ACKNOWLEDGEMENTS}

The authors thank Dr. Abdel- Aziz MS, microbiological Department, national research Centre, Dokki, Giza, Egypt, for assistance with the microbiological assays.

\section{CONCLUSION}

Chitosan/MMT and PVA/AgNPs nanocomposites prepared and forming membranes using freeze-thawing technique with 3 freeze cycle, each cycle has the duration of 24hr with 50:50 (W:W) composition. The properties of the prepared hydrogels were investigated and compared to the prepared hydrogel in absence of nanometals. The obtained results showed positive effect of nanoparticles especially AgNPs on swelling percent on the other hand tensile strength combined by presence of MMT nanoparticles. The surface morphology showed homogenous images for all samples not containing MMT and open pores for membrane not containing nanometals. All prepared samples containing nanoparticles showed antibacterial activity especially membranes containing AgNPs.

\section{REFERENCES}

Bhowmick S, Koul V. Assessment of PVA/silver nanocomposite hydrogel patch as antimicrobial dressing scaffold: Synthesis, characterization and biological evaluation. Materials Science and Engineering: C, 2016; 59: 109-119.

Catanzano O, D'esposito V, Acierno S, Ambrosio MR, De Caro C, Avagliano C, Russo P, Russo R, Miro A, Ungaro F, Calignano A, Formisano P,Quaglia F. Alginate-hyaluronan composite hydrogels accelerate wound healing process. Carbohydrate Polymers, 2015; 131: 407-414

Silva D, Fernandes AC, Nunes TG, Colaço R,Serro AP. The effect of albumin and cholesterol on the biotribological behavior of hydrogels for contact lenses. Acta Biomaterialia, 2015; 26: 184-194.

Pairatwachapun S, Paradee N,Sirivat A. Controlled release of acetylsalicylic acid from polythiophene/carrageenan hydrogel via electrical stimulation. Carbohydrate Polymers, 2016; 137: 214-221.

Mabry KM, Payne SZ,Anseth KS. Transcriptional profiles of valvular interstitial cells cultured on tissue culture polystyrene, on 2D hydrogels, or within 3D hydrogels. Data in Brief, 2015; 5: 959-962.

Abdel-Mohsen AM, Aly AS, Hrdina R, Montaser AS,Hebeish A. Eco-Synthesis of PVA/Chitosan Hydrogels for Biomedical Application. Journal of Polymers and the Environment, 2011; 19: 1005-1012.

Kim TH, An DB, Oh SH, Kang MK, Song HH,Lee JH. Creating stiffness gradient polyvinyl alcohol hydrogel using a simple gradual freezing-thawing method to investigate stem cell differentiation behaviors. Biomaterials, 2015; 40: 51-60.

Zhang D, Duan J, Wang D,Ge S. Effect of Preparation Methods on Mechanical Properties of PVA/HA Composite Hydrogel. Journal of Bionic Engineering, 2010; 7: 235-243.

Abdel-Mohsen AM, Aly AS, Hrdina R, Montaser AS,Hebeish A. Biomedical Textiles Through Multifunctioalization of Cotton Fabrics Using Innovative Methoxypolyethylene Glycol-N-Chitosan Graft Copolymer. Journal of Polymers and the Environment, 2012; 20: 104-16.

Hebeish AA, Ramadan MA, Montaser AS,Farag AM. Preparation, characterization and antibacterial activity of chitosan-g-poly acrylonitrile/silver nanocomposite. International Journal of Biological Macromolecules, 2014; 68: 178-184.

Hebeish AA, Ramadan MA, Krupa I, Montaser AS,Salama AA, Abdel-Aziz MS. In vitro and in vivo antibacterial potential of chitosan-gacrylonitrile silver nanocomposite against a pathogenic bacterium. Int. J. Curr. Microbiol. App. Sci., 2015; 4: 5-19.

Hebeish AA, Ramadan MA, Montaser AS,Krupa I,Farag AM. Molecular Characteristics and Antibacterial Activity of Alginate Beads Coated Chitosan Polyacrylonitrile Copolymer Loaded Silver Nanocomposite. Journal of Scientific Research and Reports, 2015; 5: 479 488.

Hebeish AA, Aly AS, Ramadan MA, Abd El-Hady MM, Montaser AS,Farag AM. Establishment of optimum conditions for preparation of silver nanoparticles using carboxymethyl chitosan. Egyptian Journal of Chemistry, 2013; 56: 241-254.

Hsu S-H, Wang M-C,Lin J-J. Biocompatibility and antimicrobial evaluation of montmorillonite/chitosan nanocomposites. Applied Clay Science, 2012; 56: 53-62. 
Abureesh MA, Oladipo AA,Gazi M. Facile synthesis of glucose-sensitive chitosan-poly(vinyl alcohol) hydrogel: Drug release optimization and swelling properties. International Journal of Biological Macromolecules, 2015; pii: S0141-8130(15)30005-2.

Liu Y, Geever LM, Kennedy JE, Higginbotham CL, Cahill PA,Mcguinness GB. Thermal behavior and mechanical properties of physically crosslinked PVA/Gelatin hydrogels. Journal of the Mechanical Behavior of Biomedical Materials, 2010; 3: 203-209.

Guo L, Yuan W, Lu Z,Li CM. Polymer/nanosilver composite coatings for antibacterial applications. Colloids and Surfaces A: Physicochemical and Engineering Aspects, 2013; 439: 69-83.

Chhatri A, Bajpai J, Bajpai AK, Sandhu SS, Jain N,Biswas J. Cryogenic fabrication of savlon loaded macroporous blends of alginate and polyvinyl alcohol (PVA). Swelling, deswelling and antibacterial behaviors. Carbohydrate Polymers, 2011; 83: 876-882.

Tong X, Zheng J, Lu Y, Zhang Z,Cheng H. Swelling and mechanical behaviors of carbon nanotube/poly(vinyl alcohol) hybrid hydrogels. Materials Letters, 2007; 61: 1704-1706.

Lavorgna M, Attianese I, Buonocore GG, Conte A, Del Nobile MA, Tescione F,Amendola E. MMT-supported Ag nanoparticles for chitosan nanocomposites: Structural properties and antibacterial activity. Carbohydrate Polymers, 2014; 102: 385-392.

\section{How to cite this article:}

Montaser AS. Physical, Mechanical and Antimicrobial Evaluations of Physically Crosslinked PVA/Chitosan Hydrogels Containing Nanoparticles. J App Pharm Sci, 2016; 6 (05): 001-006. 